\title{
The Structure and Kinematics of Envelope around Red Supergiant AH Sco Traced by $\mathrm{SiO}$ Masers
}

\author{
X. Chen ${ }^{1}$ and Z.-Q. Shen ${ }^{1}$ \\ ${ }^{1}$ Shanghai Astronomical Observatory, 80 Nandan Road, Shanghai 200030, PR China \\ email: chenxi, zshen@shao.ac.cn
}

\begin{abstract}
Observations of $43 \mathrm{GHz} v=1, J=1-0 \mathrm{SiO}$ masers in the circumstellar envelope of the M-type semi-regular variable star AH Sco were performed with the Very Long Baseline Array (VLBA) at 2 epochs in March 2004. These high-resolution VLBA images reveal that the distribution of $\mathrm{SiO}$ masers is roughly on a persistent elliptical ring with the lengths of the major and minor axes of about 18.5 and 15.8 mas, respectively, along a position angle of $150^{\circ}$. The 3 -dimensional kinematics model-fitting for proper motions and spatial distributions of maser features clearly indicates that the $\mathrm{SiO}$ maser shell around $\mathrm{AH}$ Sco was undergoing an overall contraction to the star at a velocity of $15 \mathrm{~km} \mathrm{~s}^{-1}$ at a distance of $2.26 \mathrm{kpc}$ to AH Sco due to the gravitation of the central star.
\end{abstract}

Keywords. (Stars:) circumstellar matter - masers - stars: individual (AH Sco)

\section{VLBA observations}

Late type stars often exhibit circumstellar maser emission in molecules e.g. $\mathrm{OH}, \mathrm{H}_{2} \mathrm{O}$, and $\mathrm{SiO}$. The interferometric observations of these masers would be useful in determining the structure and kinematics of the circumstellar envelop (CSE) and understanding the physical circumstance and mass loss procedure for late type stars. In this paper, we present the first VLBI maps of $43 \mathrm{GHz} v=1, J=1-0 \mathrm{SiO}$ maser emission toward red supergiant $\mathrm{AH}$ Scorpii (AH Sco). The observations were performed at two epochs on March 8, 2004 (epoch A) and March 20, 2004 (epoch B) with the VLBA. The data were recorded in left circular polarization in an $8 \mathrm{MHz}$ band and correlated with the FX correlator in Socorro, New Mexico. The correlator output data had 256 spectral channels, corresponding to a velocity resolution of $0.22 \mathrm{~km} \mathrm{~s}^{-1}$.

\section{The spatial structure of the $\mathrm{SiO}$ masers}

The high resolution VLBI images reveal a persistent elliptical structure of $\mathrm{SiO}$ masers around AH Sco during an interval of 12 days (Fig. 1). We characterized this morphology by performing a least-squares fit of an ellipse to the distribution of masers weighted by the flux density of each feature for each of two epochs. The lengths of the major and minor axes were found to be 18.6 and 15.7 mas for epoch A, and 18.4 and 15.9 mas for epoch B, respectively, with the major axis of the ellipse oriented similarly at $150^{\circ}$ at both epochs. We also notice that the red-shifted $\mathrm{SiO}$ masers lie slightly closer to the center than the blue-shifted masers.

\section{The kinematics of the $\mathrm{SiO}$ masers}

By identifying the matched common maser features that appeared in both epochs, we were able to estimate their proper motions (Fig. 2). We can see that the maser shell shows an overall contraction toward the central star. We have made a 3-dimensional kinematics model-fitting for spatial distribution and proper motion of $\mathrm{SiO}$ maser features (see details 

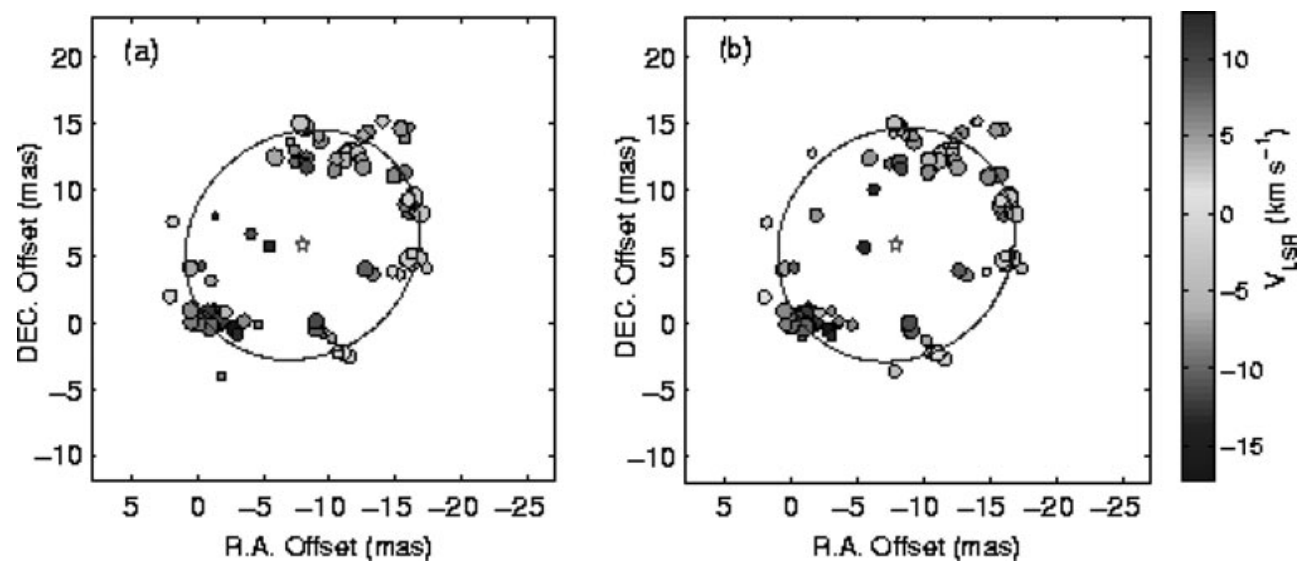

Figure 1. VLBI images of $43 \mathrm{GHz} v=1, J=1-0 \mathrm{SiO}$ maser emission toward AH Sco at two epochs. The ellipse indicates the least-squares fit to the maser distribution for each epoch. The fitted center of ellipse model is marked by the red star.

in Chen \& Shen 2008). The 3-dimensional kinematics model suggested that the SiO maser shell was undergoing an overall contraction to the star at a velocity of $15 \mathrm{~km} \mathrm{~s}^{-1}$ at a distance of $2.26 \mathrm{kpc}$ to $\mathrm{AH}$ Sco due to the gravitation of the central star.

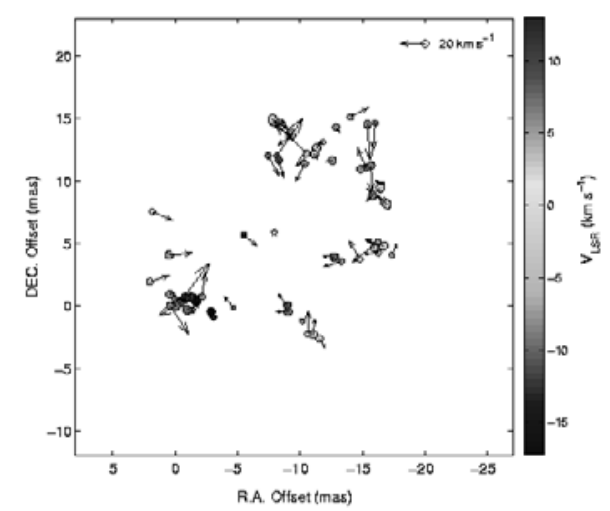

Figure 2. Distribution of proper motion velocity vectors of the matched maser features at a distance of $2.26 \mathrm{kpc}$.
Interestingly, the optical phase at which the $\mathrm{SiO}$ maser shell around the red supergiants contracts is nearly the same as that seen in Mira variables: red supergiant $\mathrm{AH} \operatorname{Sco}(\phi \approx 0.55$; Chen \& Shen 2008); red supergiant VX Sgr $(\phi=$ 0.75-0.80; Chen et al. 2006), Mira variable R Aqr $(\phi=0.78-0.04$; Boboltz, Diamond \& Kemball 1997), Mira variable TX Cam $(\phi=0.50-0.65$; Diamond \& Kemball 2003). This infers that the contraction of the $\mathrm{SiO}$ maser shell would occur during an optical stellar phase of 0.5-1, which agrees with the theoretical kinematical model results of Humphreys et al. (2002).

Acknowledgement. This work was supported in part by the National Natural Science Foundation of China (grants 10573029, 10625314, and 10633010) and the Knowledge Innovation Program of the Chinese Academy of Sciences (Grant No. KJCX2-YW-T03), and sponsored by the Program of Shanghai Subject Chief Scientist (06XD14024) and the National Key Basic Research Development Program of China (No. 2007CB815405). X. Chen also thanks the support by the Knowledge Innovation Program of the Chinese Academy of Sciences.

\section{References}

Boboltz, D. A., Diamond, P. J., \& Kemball, A. J. 1997, ApJ, 487, L147

Chen, X. \& Shen, Z.-Q. 2008, ApJ, (in press); astro-ph/08031690

Chen, X., Shen, Z.-Q., Imai, H., et al. 2006, ApJ, 640, 982

Diamond, P. J. \& Kemball, A. J. 2003, ApJ, 599, 1372

Humphreys, E. M. L., Gray, M. D., Yates, J. A., et al. 2002, A\& $A, 386,256$ 